PROCEEDINGS OF THE

AMERICAN MATHEMATICAL SOCIETY

Volume 123, Number 4, April 1995

\title{
INITIAL VALUE PROBLEM AND GLOBAL HOMEOMORPHISMS
}

\author{
MENG SU
}

(Communicated by Palle E. T. Jorgensen)

\begin{abstract}
Some theorems about the existence of solutions of nonlinear operator equation and the global homeomorphism in abstract space by the global existence theorem of an initial value problem are given. They are generalizations of some well-known results in these areas.
\end{abstract}

\section{INTRODUCTION}

The existence of solutions of a nonlinear operator equation by means of a differential equation in abstract spaces is proved effectively in some cases. For example, Browder applied the results of nonlinear evolution equations and obtained the general existence theorems for solutions of the nonlinear accretive operator-equation (see [1, $\S 10 ; 2])$. A global existence theorem of an initial value problem (IVP) was applied by Su and Xu [9] to obtain the global existence of solutions of a nonlinear operator equation.

In this paper, we apply the most general theorem of global existence of solutions of a IVP with the Layapunov-like function (see [5]) and obtain some general results for the existence of solutions of a nonlinear operator equation and the global homeomorphism between two Banach spaces. In particular, we obtain an interesting global homeomorphism theorem in Hilbert space.

In $\S 2$, we give preliminaries concerning some concepts, properties, and relations. We show in $\S 3$ some existence results for an abstract differential equation. These results are then used in the last section to obtain the main theorems about existence of solutions of a nonlinear equation and global homeomorphism which is the generalization of several well-known theorems due to Hadamard and Lévy $[7,8]$ and Palais [6].

\section{Preliminary}

Throughout the paper we use the following notation. We denote by $R$ the field of real numbers and by $R_{+}$the set $\{x \in R: x>0\}$. If $E$ and $F$ are two Banach spaces, then $\operatorname{Isom}(E, F)$ denotes the set of all linear continuous isomorphisms of $E$ onto $F$.

Received by the editors July 7, 1993.

1991 Mathematics Subject Classification. Primary 34G20, 47H15.

Key words and phrases. Initial value problem, Lyapunov-like function, global existence of solution, nonlinear operator equation, global homeomorphism. 
For every $x, y \in E$, we define

$$
\begin{aligned}
& {[x, y]_{ \pm}=\lim _{t \rightarrow 0 \pm} \frac{1}{t}(\|x+t y\|-\|x\|)} \\
& (x, y)_{ \pm}=\frac{1}{2} \lim _{t \rightarrow 0 \pm} \frac{1}{t}\left(\|x+t y\|^{2}-\|x\|^{2}\right) .
\end{aligned}
$$

These definitions may be found in [5]. The semi-inner product $(\cdot, \cdot)_{ \pm}$has some of the properties of an inner product. If $E$ is a Hilbert space, it is just the inner product $\langle\cdot, \cdot\rangle$. Here we list several properties of such directional derivatives. They will be used in the following

Lemma 1 [5]. For any $x, y \in E$, we have

(i) $\left|[x, y]_{ \pm}\right| \leq\|y\|$,

(ii) $\left|(x, y)_{ \pm}\right| \leq\|x\| \cdot\|y\|$,

(iii) $(x, y)_{ \pm}=\|x\|[x, y]_{ \pm}$.

If $x: R \rightarrow E$, such that $x_{ \pm}^{\prime}(t)$ (the right and left derivative of $x(t)$ ) exists for some $t \in R$, and $m(t)=\|x(t)\|$ and $n(t)=\frac{1}{2}\|x(t)\|^{2}$, then

(iv) $m_{ \pm}^{\prime}(t)$ exist and $m_{ \pm}^{\prime}(t)=\left[x(t), x_{ \pm}^{\prime}(t)\right]_{ \pm}$,

(v) $n_{ \pm}^{\prime}(t)$ exist and $n_{ \pm}^{\prime}(t)=\left(x(t), x_{ \pm}^{\prime}(t)\right)_{ \pm}$.

Consider the mapping $f: E \rightarrow F$. Suppose that $f^{\prime}(x) \in \operatorname{Isom}(E, F)$ for every $x \in E$. Then the following initial value problem with parameters $v$ in $F$ which is called Wazewski's differential equation (see [3])

$$
x^{\prime}(t)=f^{\prime}(x)^{-1} v, \quad x(0)=x_{0}
$$

is equivalent to the equality

$$
f(x(t))=f\left(x_{0}\right)+v t .
$$

Let $v=y-f\left(x_{0}\right), y \in F, t=1$. Then (2) is the nonlinear equation

$$
f(x)=y \text {. }
$$

Obviously, $x(1)$ is the solution of equation (3) provided the differential equation (1) has a global solution $x(t)$ on $[0,1]$.

It is our main objective to find general conditions to ensure the existence of solutions of nonlinear equation (3). Wazewski's equation (1) can be regarded as an auxiliary ordinary differential equation of the operator $f$.

The relation between equation (3) and IVP (1) is as follows.

Lemma 2. Suppose $f: E \rightarrow F$ is a continuously differentiable map and $f^{\prime}(x) \in$ $\operatorname{Isom}(E, F)$ for every $x \in E$. If there exists $x_{0} \in E$ for $v=y-f\left(x_{0}\right)$ such that the IVP (1) has a solution $x(t)$ on $[0,1]$, then we have

(i) there exists a solution of equation (3),

(ii) furthermore, if for any fixed $x_{0} \in E, v \in F$, such that IVP (1) has a solution $x(t)$ on $[0,1]$, then $f$ is a global homeomorphism.

Proof. Conclusion (i) is obvious. We prove (ii) in detail.

For any $y \in E$, set $v=y-f\left(x_{0}\right)$; we have that $f$ is surjective by (i).

Thus it remains only to prove that $f$ is injective. Suppose that $x_{1}$ and $x_{2}$ are solutions of equation (3), and let $\tilde{x}(\lambda)=\lambda x_{1}+(1-\lambda) x_{2}, 0 \leq \lambda \leq 1$. Then

$$
\tilde{x}(0)=x_{2}, \quad \tilde{x}(1)=x_{1} .
$$


It follows from the condition of (ii) and the local homeomorphism of $f$ that Wazewski's problem

$$
x^{\prime}(t)=f^{-1}(x) v_{\lambda}, \quad x(0, \lambda)=\tilde{x}(\lambda)
$$

has a solution for any $v_{\lambda} \in F$. Set $v_{\lambda}=y-f(\tilde{x}(\lambda))$. The solution $x(t, \lambda)$ of (4) satisfies

$$
f(x(t, \lambda))=f(\tilde{x}(\lambda))+t(y-f(\tilde{x}(\lambda)), \quad x(0, \lambda)=\tilde{x}(\lambda) \quad \text { on }[0,1] .
$$

Note that $f(x(t, 0))=f(x(t, 1))=y$ and $x(0,0)=x_{2}$ and $x(0,1)=x_{1}$. By a local homeomorphism of $f$ at $x_{1}$ and $x_{2}$, we have

$$
x(t, 0) \equiv x_{2}, \quad x(t, 1) \equiv x_{1} \quad \text { for } 0 \leq t \leq 1 .
$$

However, by (5) $f(x(1, \lambda)) \equiv y$ for $0 \leq \lambda \leq 1$. Again by local homeomorphism of $f$, we get that $x(1, \lambda)$ is a constant over $0 \leq \lambda \leq 1$. Therefore, $x_{2}=x(1,0)=x(1,1)=x_{1}$ and the uniqueness is verified. The proof is complete.

\section{Global EXISTENCE OF AN INITIAL VALUE PROBLEM}

In this section, we are concerned with the global existence of solutions of the initial value problem (IVP):

$$
x^{\prime}(t)=f(t, x), \quad x\left(t_{0}\right)=x_{0},
$$

where $f:\left[t_{0}, T\right] \times E \rightarrow E$ is a continuous function ( $T$ may be the infinity $+\infty)$. Write $J=\left[t_{0}, T\right]$.

A general condition with Lyapunov-like function is given to assert the global existence of solutions. We denote by $\Omega$ the set of all Lyapunov-like functions $V(t, x)$ that satisfies the conditions:

(i) $V \in C\left[J \times E, R_{+}\right], V$ is locally Lipschitzian in $x$;

(ii) $V(t, x) \rightarrow \infty$ as $\|x\| \rightarrow \infty$ uniformly for $[0, T]$.

The following theorem is cited from [5].

Theorem 1. Assume that:

(A1) $f \in C[J \times E, E], f$ is bounded on bounded sets, and for any $\left(t_{0}, x_{0}\right) \in$ $J \times E$ there exists a local solution for the problem (6).

(A2) $V \in \Omega$ and for $(t, x) \in J \times E$

$$
D^{+} V(t, x) \equiv \lim _{h \rightarrow 0} \frac{1}{h}[V(t+h, x+h f(t, x))-V(t, x)] \leq g(t, V(t, x)),
$$

where $g \in C[J \times E, R]$. tion

(A3) The maximal solution $r(t)=r\left(t, t_{0}, u_{0}\right)$ of the scalar differential equa-

$$
u^{\prime}=g(t, u), \quad u\left(t_{0}\right)=u_{0}>0
$$

exists on $J$ and is positive.

Then for every $x_{0} \in E$ such that $V\left(t_{0}, x_{0}\right) \leq u_{0}$, the problem (6) has a solution $x(t)$ on $J$ which satisfies the estimate

$$
V(t, x(t)) \leq r(t), \quad t \in J .
$$

Several corollaries are given by setting $V(x)=\left\|x-x_{1}\right\|$ and $\left\|x-x_{1}\right\|^{2}$, where $x_{1} \in E$. 
Corollary 1. Assume that $f \in C[J \times E, E], f$ is bounded on bounded sets, and for any $\left(t_{0}, x_{0}\right) \in J \times E$ there exists a local solution for the problem (6). We also assume that there exists $x_{1} \in E$ and for $(t, x) \in J \times E$,

$$
\left[x-x_{1}, f(t, x)\right]_{+} \leq g\left(t,\left\|x-x_{1}\right\|\right),
$$

where $g \in C\left[J \times R_{+}, R\right]$ satisfies condition (A3).

Then for every $x_{0} \in E$ such that $\left\|x_{0}-x_{1}\right\| \leq u_{0}$ the problem (6) has a solution $x(t)$ on $J$ which satisfies the estimate

$$
\left\|x(t)-x_{1}\right\| \leq r(t), \quad t \in J .
$$

Proof. Define $V(x)=\left\|x-x_{1}\right\|$. Obviously $V(x) \in \Omega$. By Lemma 1 and inequality $(8)$ we have that

$$
D^{+} V(x)=\left[x(t)-x_{1}, x^{\prime}(t)\right]_{+}=\left[x(t)-x_{1}, f(t, x(t))\right]_{+} \leq g(t, V(x)) .
$$

Hence, the proof is immediately completed by Theorem 1 .

Corollary 2. Assume that $f$ satisfies all conditions in Corollary 1 except (8) and that there exists $x_{1} \in E$ such that

$$
\left(x-x_{1}, f(t, x)\right)_{+} \leq \frac{1}{2} g\left(t,\left\|x-x_{1}\right\|^{2}\right)
$$

where $g \in C\left[J \times R_{+}, R\right]$ satisfies (A3).

Then for every $x_{0} \in E$ such that $\left\|x_{0}-x_{1}\right\|^{2} \leq u_{0}$ problem (6) has a solution $x(t)$ on $J$ and satisfies the estimate $\left\|x(t)-x_{1}\right\|^{2} \leq r(t), t \in J$.

Proof. Letting $V(x)=\left\|x-x_{1}\right\|^{2} \in \Omega$, by Lemma 1 we have that

$$
D^{+} V(x)=2\left(x(t)-x_{1}, f(t, x)\right)_{+} \leq g(t, V(x)) .
$$

Hence, by Theorem 1, we have proved this corollary.

Remark 1. By Lemma 1, inequality (8) may be replaced by a more strict condition:

$$
\|f(t, x)\| \leq g\left(t,\left\|x-x_{1}\right\|\right),
$$

where $g$ is positive and satisfies (A3).

Furthermore, we have the generalization of Wintner's theorem in abstract space by setting $x_{1}=0$. You may find it in [5].

Remark 2. If $E$ is a Hilbert space, inequality (9) becomes

$$
\left\langle x-x_{1}, f(t, x)\right\rangle \leq \frac{1}{2} g\left(t,\left\|x-x_{1}\right\|^{2}\right),
$$

where $\langle\cdot, \cdot\rangle$ is the inner product (see $[4$, Theorem $C]$ ).

\section{EXISTENCE OF SOLUTION, GLOBAL HOMEOMORPHISM}

In this section, we discuss the nonlinear operator equation (3) by applying the results of $\S 3$ to the Wazewski equation (1).

In order to prove the main theorem, we give a lemma in advance.

Lemma 3. Suppose that $\lambda(\cdot)$ and $\omega(\cdot) \in C\left[J, R_{+}\right]$and satisfy the inequality

$$
\int_{u_{0}}^{+\infty} \frac{d u}{\omega(u)}>\int_{t_{0}}^{T} \lambda(\tau) d \tau
$$


Then there exists a global solution of the initial value problem

$$
u^{\prime}=\omega(u) \lambda(t), \quad u\left(t_{0}\right)=u_{0}
$$

on $J$.

Proof. As $\omega(\cdot), \lambda(\cdot)$ are continuous functions, IVP (13) has a local solution $u(t)$ by Peano's theorem. Since $\omega(u)>0,(13)$ implies that for any solution $u(t)$

$$
\int_{u_{0}}^{u(t)} \frac{d u}{\omega(u)}=\int_{t_{0}}^{t} \lambda(\tau) d \tau
$$

By the extension theorem of a solution (see [3]), $u(t)$ can be extended over the interval of existence $\left[t_{0}, T\right]$ because the solution $u(t)$ can fail to exist on $\left[t_{0}, T\right]$ only if it exists on some interval $\left[t_{0}, \delta\right)$ and satisfies $u(t) \rightarrow \infty$ as $t \rightarrow \delta(\leq T)$. If this is the case, however, $t \rightarrow \delta$ in (14) gives a contradiction with inequality (12). This completes the proof.

The main theorem is the following.

Theorem 2. Suppose the mapping $f$ satisfies:

(B1) $f \in C^{1}[E, F]$ and $f^{\prime}(x) \in \operatorname{Isom}(E, F)$.

(B2) $\sup _{\|x\| \leq r}\left\|f^{\prime}(x)\right\|<+\infty \quad \forall r: 0<r<+\infty$.

(B3) There exist $x_{0}$ and a function $U: E \rightarrow R_{+}, U \in \Omega$, for $v=y-f\left(x_{0}\right)$ satisfying

$$
D^{+} U(x)=\lim _{h \rightarrow 0} \frac{1}{h}\left[U\left(x+h f^{\prime}(x)^{-1} v\right)-U(x)\right] \leq h_{v}(U(x)),
$$

where $h_{v} \in C\left[R_{+}, R_{+}\right]$satisfies the inequality

(B4) $\int_{U\left(x_{0}\right)}^{+\infty} \frac{d \sigma}{h_{v}(\sigma)}>1$.

Then we have

(i) equation (3) has a solution,

(ii) if (B4) is replaced by

(B5) $\int_{U\left(x_{0}\right)}^{+\infty} \frac{d \sigma}{h_{v}(\sigma)}=+\infty$, for all fixed $x_{0} \in E, v \in F$,

then $f$ is a global homeomorphism.

Proof. Consider the IVP

$$
x^{\prime}(t)=f^{\prime}(x)^{-1} v, \quad x(0)=x_{0},
$$

where $v=y-f\left(x_{0}\right)$.

By condition (B4), Lemma 3 implies that the scalar initial value problem

$$
u^{\prime}(t)=h_{v}(u), \quad u(0)=U\left(x_{0}\right)
$$

has a solution on $[0,1]$.

It is easy to verify that all the conditions in Theorem 1 are satisfied for IVP (15) under the assumptions (B1)-(B4). Hence, there exists a solution $x(t)$ of (15) on $J=[0,1]$. By Lemma $2, x(1)$ is the solution of (3).

We next prove (ii). (B4) holds for all fixed $x_{0} \in E, v \in F$ under the assumption (B5). Hence, IVP (15) has the solution $x(t)$ on $[0,1]$ for any $x_{0}$, $v$. Using Lemma 2, we immediately obtain (ii).

The following theorem is from Corollaries 1 and 2 and Lemma 2. We omit the proof since it is the same as above. 
Theorem 3. Suppose the mapping $f$ has properties (B1), (B2) and that there exist $x_{0}, x_{1} \in E$ satisfy either one of the following conditions:

(C1) $\left[x-x_{1}, f^{\prime}(x)^{-1} v\right]_{+} \leq h_{v}\left(\left\|x-x_{1}\right\|\right)$, where $h_{v}(\cdot) \in C\left[R_{+}, R_{+}\right]$satisfies

$$
\int_{\left\|x_{0}-x_{1}\right\|}^{+\infty} \frac{d \sigma}{h_{v}(\sigma)}>1
$$
isfies

(C2) $\left(x-x_{1}, f^{\prime}(x)^{-1} v\right)_{+} \leq \frac{1}{2} k_{v}\left(\left\|x-x_{1}\right\|^{2}\right)$, where $k_{v}(\cdot) \in C\left[R_{+}, R_{+}\right]$sat-

$$
\int_{\left\|x_{0}-x_{1}\right\|^{2}}^{+\infty} \frac{d \sigma}{k_{v}(\sigma)}>1
$$

Then

(i) equation $f(x)=y$ has a solution;

(ii) if (16) or (17) is replaced by $\int_{\left\|x_{0}-x_{1}\right\|}^{+\infty} \frac{d \sigma}{h_{v}(\sigma)}=+\infty$ or $\int_{\left\|x_{0}-x_{1}\right\|^{2}}^{+\infty} \frac{d \sigma}{k_{v}(\sigma)}=+\infty$ for all $x_{0} \in E, v \in F$, then $f$ is a global homeomorphism.

We find that some known results are the consequences of above theorems.

Theorem 4 [9]. Suppose that $f$ satisfies (B1) and

$$
\left\|f^{\prime}(x)^{-1}\right\| \leq \omega(\|x\|) \quad \forall x \in E,
$$

where $\omega: R_{+} \rightarrow R_{+}$is a continuous function.

Suppose also that there exists an $x_{0} \in E$ such that

$$
\int_{\left\|x_{0}\right\|}^{+\infty} \frac{d \sigma}{\omega(\sigma)}>\left\|f\left(x_{0}\right)-y\right\|
$$

Then equation $f(x)=y$ has a solution.

Proof. Suppose $f\left(x_{0}\right) \neq y$, and let $x_{1}=0, h_{v}(s)=\left\|f\left(x_{0}\right)-y\right\| \omega(s)$. By Lemma 1 and (18), (19), we have

$$
\begin{gathered}
{\left[x, f^{\prime}(x)^{-1} v\right]_{+} \leq\left\|f^{\prime}(x)^{-1}\right\| \cdot\|v\| \leq\left\|f\left(x_{0}\right)-y\right\| \omega(\|x\|)=h_{v}(\|x\|),} \\
\int_{\left\|x_{0}\right\|}^{+\infty} \frac{d \tau}{h_{v}(\tau)}>1 .
\end{gathered}
$$

Thus condition (C1) holds. (B2) is satisfied according to (18). By Theorem 3, we complete the proof.

Theorem $5[7,8]$. Let $f$ satisfy (B1) and $\left\|f^{\prime}(x)^{-1}\right\| \leq \omega(\|x\|)$ for all $x \in E$, where $\omega(\cdot): R_{+} \rightarrow R_{+}$is a continuous function and

$$
\int_{\left\|x_{0}\right\|}^{+\infty} \frac{d \sigma}{\omega(\sigma)}=+\infty
$$

Then $f$ is a homeomorphism from $E$ onto $F$.

This theorem can be proved easily by Theorem 3(ii).

Theorem 6 [6]. If $f$ satisfies (B1), (B2) and is coercive, i.e., $\|f(x)\| \rightarrow+\infty$ as $\|x\| \rightarrow+\infty$, then $f$ is a global homeomorphism.

Proof. Consider $x^{\prime}(t)=f^{\prime}(x)^{-1} v, x(0)=x_{0}, v \in E$. Define $K(x)=\|f(x)\|$, where $f$ is coercive; thus, $K(x) \in \Omega$ and

$$
D^{+} K(x(t))=\left[f(x(t)), f^{\prime}(x) x^{\prime}(t)\right]_{+}=[f(x(t)), v]_{+} \leq\|v\|,
$$


where $\|v\|$ is a positive constant which satisfies condition (B5). Therefore, by Theorem 2(ii) $f$ is a global homeomorphism.

Finally we give a global theorem in Hilbert space. It is deduced from Theorem 3 and is a generalization of Hadamard-Levy's Theorem (Theorem 5) in Hilbert space. We denote by $\left[f^{\prime}(x)^{-1}\right]^{*}$ the dual operator of $f^{\prime}(x)^{-1} \in L(F, E)$.

Theorem 7. Suppose that $E$ is a Hilbert space and $f$ satisfies conditions (B1), (B2). If there exists $x_{1} \in E$ such that

$$
\left\|\left[f^{\prime}(x)^{-1}\right]^{*}\left(x-x_{1}\right)\right\| \leq \omega\left(\left\|x-x_{1}\right\|^{2}\right),
$$

where $\omega$ satisfies conditions in Theorem 5 , then $f$ is a global homeomorphism from $E$ onto $F$.

Proof. Since $E$ is a Hilbert space, we have

$$
\begin{aligned}
\left(x-x_{1}, f^{\prime}(x)^{-1} v\right)_{+} & =\left\langle x-x_{1}, f^{\prime}(x)^{-1} v\right\rangle=\left\langle\left[f^{\prime}(x)^{-1}\right]^{*}\left(x-x_{1}\right), v\right\rangle \\
& \leq \|\left[f^{\prime}(x)^{-1}\right]^{*}\left(x-x_{1}\|\cdot\| v\left\|\leq \omega\left(\left\|x-x_{1}\right\|^{2}\right) \cdot\right\| v \| .\right.
\end{aligned}
$$

Let $k_{v}(s)=2 \omega(s)\|v\|$. Condition (C2) in Theorem 3 holds for any $\|v\| \neq 0$ by inequality (20). Therefore, $f$ is a global homeomorphism by Theorem 3(ii).

Remark 3. Inequality (21) may be replaced by the following inequality which asserts that $(\mathrm{C} 1)$ holds, and the result of Theorem 7 remains:

There exists $x_{1} \in E$ that satisfies

$$
\left\|\left[f^{\prime}(x)^{-1}\right]^{*}\left(x-x_{1}\right)\right\| \leq\left\|x-x_{1}\right\| \psi\left(\left\|x-x_{1}\right\|\right),
$$

where $\psi: R_{+} \rightarrow R_{+}$and $\int^{+\infty} \frac{d \tau}{\psi(\tau)}=+\infty$.

It is easily known that (22) is equivalent to (21), only by the substitution $\omega(s)=\sqrt{s} \psi(\sqrt{s})$.

Remark 4. In the particular important Euclidean space, inequality (21) or (22) may be written in the following forms:

$$
\left\|\left(x-x_{1}\right) J(x)^{-1}\right\| \leq \omega\left(\left\|x-x_{1}\right\|^{2}\right)
$$

or

$$
\left\|\left(x-x_{1}\right) J(x)^{-1}\right\| \leq\left\|x-x_{1}\right\| \psi\left(\left\|x-x_{1}\right\|\right),
$$

where $J(x)$ is the Jacobian of $f(x)$.

\section{REFERENCES}

1. F. E. Browder, Nonlinear operators and nonlinear equations of evolution in Banach spaces, Proc. Sympos. Pure Math., vol. 18, Part II, Amer. Math. Soc., Providence, RI, 1976.

2. K. Deimling, Ordinary differential equations in Banach spaces, Lecture Notes in Math., vol. 596, Springer-Verlag, New York, 1976.

3. P. Hartman, Ordinary differential equations, second ed., Birkhäuser, Boston, MA, 1982.

4. T. Kato, Nonlinear equations of evolution in Banach spaces, Proc. Sympos. Pure Math., vol. 45, Part 2, Amer. Math. Soc., Providence, RI, 1986, pp. 9-23.

5. V. Lakshmikantham and S. Leela, Nonlinear differential equations in abstract spaces, Pergamon Press, New York, 1981.

6. R. S. Palais, Natural operations on differential forms, Trans. Amer. Math. Soc. 92 (1959), 125-141. 
7. R. Plastock, Homeomorphisms between Banach spaces, Trans. Amer. Math. Soc. 200 (1974), 169-183.

8. M. Radulescu and S. Raduelscu, Global inversion theorems and applications to differential equations, Nonlinear Anal. 4 (1980), 951-965.

9. M. Su and H. K. Xu, solutions of nonlinear operator equations in Banach spaces with application, Nonlinear Anal. 22 (1994), 857-865.

Department of Computer Science, East China University of Science and Technology, ShaNGhai 200237, China 